\title{
Photodynamic Therapy for Chorioretinal Diseases: A Practical Approach
}

Elon H. C. van Dijk · Thomas J. van Rijssen · Yousif Subhi •

Camiel J. F. Boon

Received: March 13, 2020 / Published online: April 11, 2020

(c) The Author(s) 2020, corrected publication 2021

\section{ABSTRACT}

Photodynamic therapy (PDT) using verteporfin (Visudyne ${ }^{\circledR}$; Bausch + Lomb) is a treatment that is widely used to elicit cell and tissue death. In ophthalmology, PDT targets choroidal vascular abnormalities and induces selective occlusion of vessels. PDT was originally used in combination with full-dose verteporfin to treat neovascular age-related macular degeneration.

Digital Features To view digital features for this article go to https://doi.org/10.6084/m9.figshare.12063741.

E. H. C. van Dijk $(\bowtie)$. T. J. van Rijssen .

C. J. F. Boon

Department of Ophthalmology, Leiden University

Medical Center, Leiden, The Netherlands

e-mail: ehcvandijk@lumc.nl

Y. Subhi

Department of Ophthalmology, Zealand University

Hospital, Roskilde, Denmark

Y. Subhi

Department of Ophthalmology, Rigshospitalet,

Glostrup, Denmark

C. J. F. Boon

Amsterdam University Medical Centers, University

of Amsterdam, Amsterdam, The Netherlands
Since the introduction of treatment with vascular endothelial growth factor receptor inhibitors, the clinical targets of PDT have shifted to other chorioretinal conditions, such as central serous chorioretinopathy, polypoidal choroidal vasculopathy, and choroidal hemangioma. In recent years, clinical studies have facilitated the optimization of treatment outcomes through changes in protocols, including the introduction of reduced treatment settings, such as PDT with half-dose verteporfin and half-fluence PDT. Here, we review PDT and its use for chorioretinal diseases from a practical perspective.

Keywords: Central serous chorioretinopathy; Choroidal hemangioma; Photodynamic therapy; Polypoidal choroidal vasculopathy 


\section{Key Summary Points}

Photodynamic therapy (PDT) plays an important role in the treatment of several chorioretinal diseases, such as central serous chorioretinopathy, polypoidal choroidal vasculopathy, and choroidal hemangioma.

The treatment of choice for chronic central serous chorioretinopathy is PDT with half-dose verteporfin or half-fluence PDT (with full-dose verteporfin).

For the treatment of polypoidal choroidal vasculopathy, PDT with both standard and reduced treatment settings can be considered.

For the treatment of choroidal hemangioma, either standard treatment settings, a bolus protocol, or a highfluence protocol can be used.

Patients should be adequately informed to avoid direct sunlight after treatment, and side effects during and after PDT are rare.

\section{INTRODUCTION}

The therapeutic use of light in medicine is not new and dates back to the ancient Egyptians and Greeks [1, 2]. The spread of Abrahamic religions that began in the fourth century resulted in both sun worship and the use of light for healing being considered to be heathen practices, with the consequence that its use for therapeutic purposes in many parts of the world was put on pause until the nineteenth century [2]. One of the pioneers who popularized light treatment in Western medicine was the Danish physician Niels Finsen, who studied the use of phototherapy against lupus vulgaris, ultimately receiving the Nobel prize in 1903 for his work in this field [2]. Subsequent research revealed that certain drug properties can be activated with the use of light; these molecules are referred to as photosensitizers and can be used in photodynamic therapy (PDT), a treatment that was first described in 1990. In the early 1990s, PDT was used to treat different types of solid tumors [3]. In PDT, a patient is given an intravenous injection of a nontoxic photosensitizer that can subsequently be locally activated using nonthermal red light. Consequently, a therapeutic effect can be achieved in a particular region $[4,5]$.

Improvements in laser technology and the development of more selective photosensitizers has resulted in PDT gaining popularity as a treatment option among ophthalmologists. One such selective photosensitizer is verteporfin (Visudyne ${ }^{\circledR} ;$ Bausch + Lomb, Laval, QB, Canada), a benzoporphyrin derivative that has a high affinity for the retinal pigment epithelium and which preferably accumulates in abnormal vascular tissue. In ophthalmology, PDT was originally used to treat subretinal neovascularization, which can occur as a late clinical manifestation of age-related macular degeneration (AMD) $[5,6]$. Following approval of PDT for treating neovascular AMD, PDT was also gradually introduced off-label at the same ('full') dose of photosensitizer for the treatment of other chorioretinal diseases, such as central serous chorioretinopathy (CSC), polypoidal choroidal vasculopathy (PCV), and choroidal hemangioma [7].

In PDT, the excitation of the photosensitizer is caused by the absorption of light. This excitation leads to local damage due to the conversion of tissue oxygen to both singlet oxygen and reactive oxygen radicals. These reactive oxygen species induce the oxidation of different structures, which in turn leads to oxidative stress and subsequently to both the apoptosis and closure of abnormal blood vessels and to stimulation of the immune system $[5,8,9]$. Three important factors need to be taken into account to induce the appropriate treatment effect: characteristics of the photosensitizer, characteristics of the involved tissue, and treatment parameters [4]. Verteporfin has a tendency to accumulate in the retinal pigment epithelium and abnormal vascular tissue and also has a high affinity for choroidal blood vessels. The latter property has been found to be 
of importance when treating diseases that are presumed to be of choroidal origin, such as CSC $[10,11]$. Verteporfin is administered intravenously and thus accumulates in the entire human body. Therefore, unprotected skin and eyes exposed to direct sunlight (which has also includes a wavelength that is similar to the $689-n m$ laser that is used during PDT) are at risk to developing side effects. For this reason, direct sunlight has to be avoided during the first few days after PDT to avoid a photosensitivity reaction, which manifests as a sunburn.

Here, we review PDT from a practical perspective and cover aspects related to the treatment protocol, safety, and information provided to the patient. This article is based on previously conducted studies and does not contain any studies with human participants or animals performed by any of the authors.

\section{PROTOCOL FOR PHOTODYNAMIC THERAPY}

Prior to a patient receiving PDT, the pupil of the eye to be treated must to be dilated, for example, by using $1.0 \%$ tropicamide and $2.5 \%$ phenylephrine. When adequate dilation is achieved, verteporfin is administered intravenously over a period of $10 \mathrm{~min}$. Historically, full-dose verteporfin $\left(6 \mathrm{mg} / \mathrm{m}^{2}\right)$ was used for PDT (full-dose PDT). However, a number of clinical studies have found that half-dose verteporfin $\left(3 \mathrm{mg} / \mathrm{m}^{2}\right)$ in PDT (half-dose PDT) usually suffices for clinical efficacy while also reducing the risk of adverse events. With halfdose PDT, the standard fluence $\left(50 \mathrm{~J} / \mathrm{cm}^{2}\right)$ and standard treatment time (83 s) are applied during the treatment. Alternative PDT options are half-fluence PDT, where the fluence is reduced to $25 \mathrm{~J} / \mathrm{cm}^{2}$ and full-dose verteporfin and the standard treatment time are applied, and halftime PDT, where the standard fluence and fulldose verteporfin are applied and the treatment time is reduced (42 s).

At exactly $15 \mathrm{~min}$ after the start of the infusion of verteporfin, an anesthetic eye drop (e.g., oxybuprocaine $0.4 \%$ ) is administered. This time interval allows for a sufficient amount of verteporfin to enter the chorioretinal tissue so that the treatment effect is maximized without increasing the risk of damage to adjacent structures. At this point, the eye can be considered ready for commencement of treatment.

For the laser treatment, typically a $\times 1.6$ magnification contact lens (e.g., a Volk ${ }^{\circledR}$ PDT lens; Volk Optical Inc., Mentor, OH, USA) is first placed on the eye to be treated, and then a $689-\mathrm{nm}$ wavelength laser is focused on the area that is to be treated. The area to be treated can be determined based on prior results from fluorescein angiography (FA) or indocyanine green angiography (ICGA).

\section{Reduced Settings: The Rationale and the Evidence}

For each particular disease entity, the balance between optimal treatment and the risk for and severity of side effects may differ. PDT using reduced treatment settings (reduced-settings PDT) may not be sufficient to achieve the desired treatment effect in some entities, but the protocol was specifically developed for treating chronic CSC in order to reduce the chance of deleterious effects [12]. In CSC, halffluence PDT has been found to be just as effective as conventional full-dose PDT [12], and in chronic CSC, half-dose PDT has been reported to be either as effective or superior to half-fluence PDT [13-15]. Dosages of $<50 \%$ have also been studied in CSC, but PDT at these lower dosages appears to be suboptimal compared to those at half-dose PDT [16, 17]. Therefore, it can be concluded that half-dose (or half-fluence) PDT should be the treatment of choice for chronic CSC [18-22]. For the treatment of PCV, half-dose PDT has also been found to have a similar efficacy as full-dose PDT when both treatments are combined with intravitreal injections with anti-vascular growth factor inhibitors (VEGFs) [23].

\section{Specific Considerations When Treating Central Serous Chorioretinopathy}

In chronic CSC, ICGA-guided PDT is performed based on the visualization of hyperfluorescent area(s) on mid-phase ICGA (approximately 

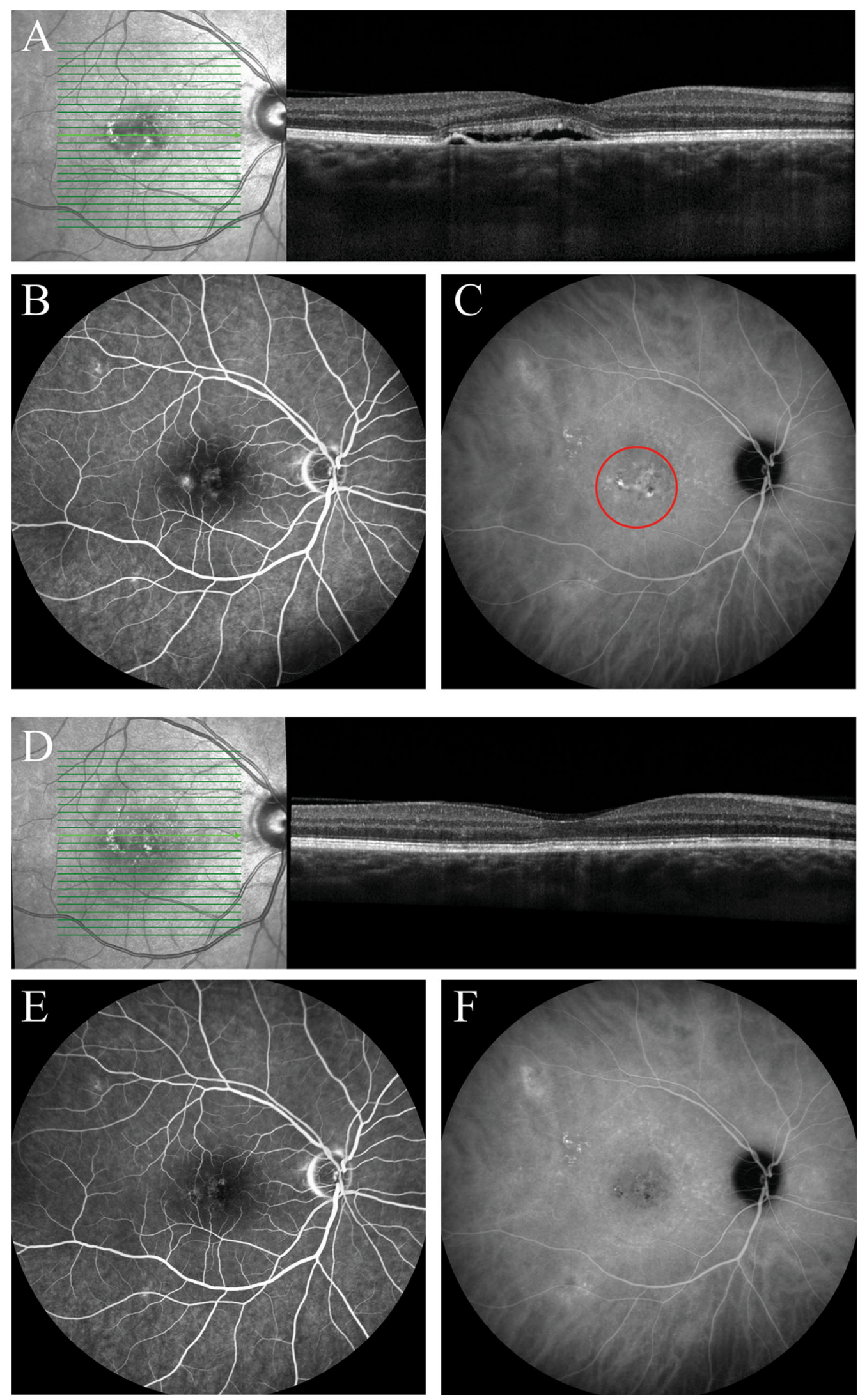
4Fig. 1 Multimodal imaging of an eye of a 55-year-old woman diagnosed with chronic central serous chorioretinopathy (CSC) and treated with half-dose photodynamic therapy (PDT). a, b Prior to initiation of half-dose PDT, foveal subretinal fluid was observed on optical coherence tomography (OCT) (a), and leakage of fluorescein and retinal pigment epithelium alterations were visible on fluorescein angiography (FA; b). c The areas of hyperfluorescence on indocyanine green angiography (ICGA), typical of chronic CSC, were included in the PDT spot (red circle). d-f Compared to the imaging results before PDT, at the visit 2 months after PDT, subretinal fluid had resolved (d), leakage of fluorescein on FA had ceased (e), and the hyperfluorescent abnormalities in the ICGA area had decreased in intensity $(\mathbf{f})$

10-15 min), which often correspond(s) to the presence of subretinal fluid (SRF) on optical coherence tomography (OCT) and leakage on mid-phase FA (approximately 3 min; Fig. 1) [20]. PDT is thought to lead to short-term hypoperfusion of the choriocapillaris and subsequently to long-term remodeling of choroidal vasculature and reduction of the increased thickness ('pachychoroid') of the large choroidal vessels, which in turn leads to a resolution of SRF leakage [10, 11]. However, promising results after FA-guided PDT in patients with chronic CSC have also been published, despite the presence of extensive choroidal abnormalities that were presumed to underlie the disease [24-28]. As these choroidal abnormalities are generally more widespread than FA abnormalities, it should be noted that FA-guided PDT of chronic CSC may not cover all of the choroidal abnormalities and could lead to undertreatment (Fig. 1) $[18,20]$. In the only available large randomized controlled trial (RCT) on chronic CSC to date, $67 \%$ of patients with chronic CSC had a complete resolution of SRF at 7-8 months of follow-up [20]. Thus, available evidence on the use of half-dose PDT in chronic CSC is considered to be level 1 due to the availability of data from this RCT [20] The effectivity of PDT may be limited in older patients and patients with hypofluorescent abnormalities on ICGA prior to treatment $[29,30]$. Alternative interventions that are available for the treatment of chronic CSC have resulted in less favorable outcomes [20, 31]. Based on published studies, ICGA-guided half-dose (or half-fluence) PDT should therefore be the treatment of choice for chronic CSC [18-22].

Chronic CSC should be treated independently of the best-corrected visual acuity (BCVA) at presentation as the prolonged presence of SRF has been found to induce irreversible damage to photoreceptors [32]. Moreover, treating patients with severe chronic CSC has been observed to lead to stable longterm BCVA [21].

Recurrence of SRF after PDT has been described in up to $20 \%$ of chronic CSC eyes $[30,33]$. A higher rate of recurrence after treatment has been found in patients with posterior cystoid retinal degeneration, no changes in intense hyperfluorescence on ICGA, poor baseline BCVA, a disrupted ellipsoid zone, and/or the presence of flat irregular detachments of the retinal pigment epithelium [34-37]. It is important to rule out the presence of a subretinal neovascularization in patients with recurrence of SRF, also using OCT angiography, if available. When no subretinal neovascularization can be found, ICGA-guided half-dose (or half-fluence) PDT should again be the treatment of choice; new contrast angiograms can be obtained to pinpoint the areas of leakage. Intravitreal injections with anti-VEGF medication should be prescribed to patients with a subretinal neovascularization [18].

\section{Specific Considerations When Treating Polypoidal Choroidal Vasculopathy}

The gold standard of PCV diagnosis requires ICGA as choroidal changes can otherwise be difficult to distinguish from, for example, neovascular AMD. Patients with PCV present with recurrent serous and/or hemorrhagic pigment epithelium detachments, with the presence of polyp-like focal hyperfluorescent areas and dilated choroidal vessels on ICGA [38]. Results from a range of clinical studies suggest that combination therapy with ICGA-guided PDT and intravitreal injections of anti-VEGF medication leads to the best visual and anatomical outcomes, as well as the highest degree of polyp 

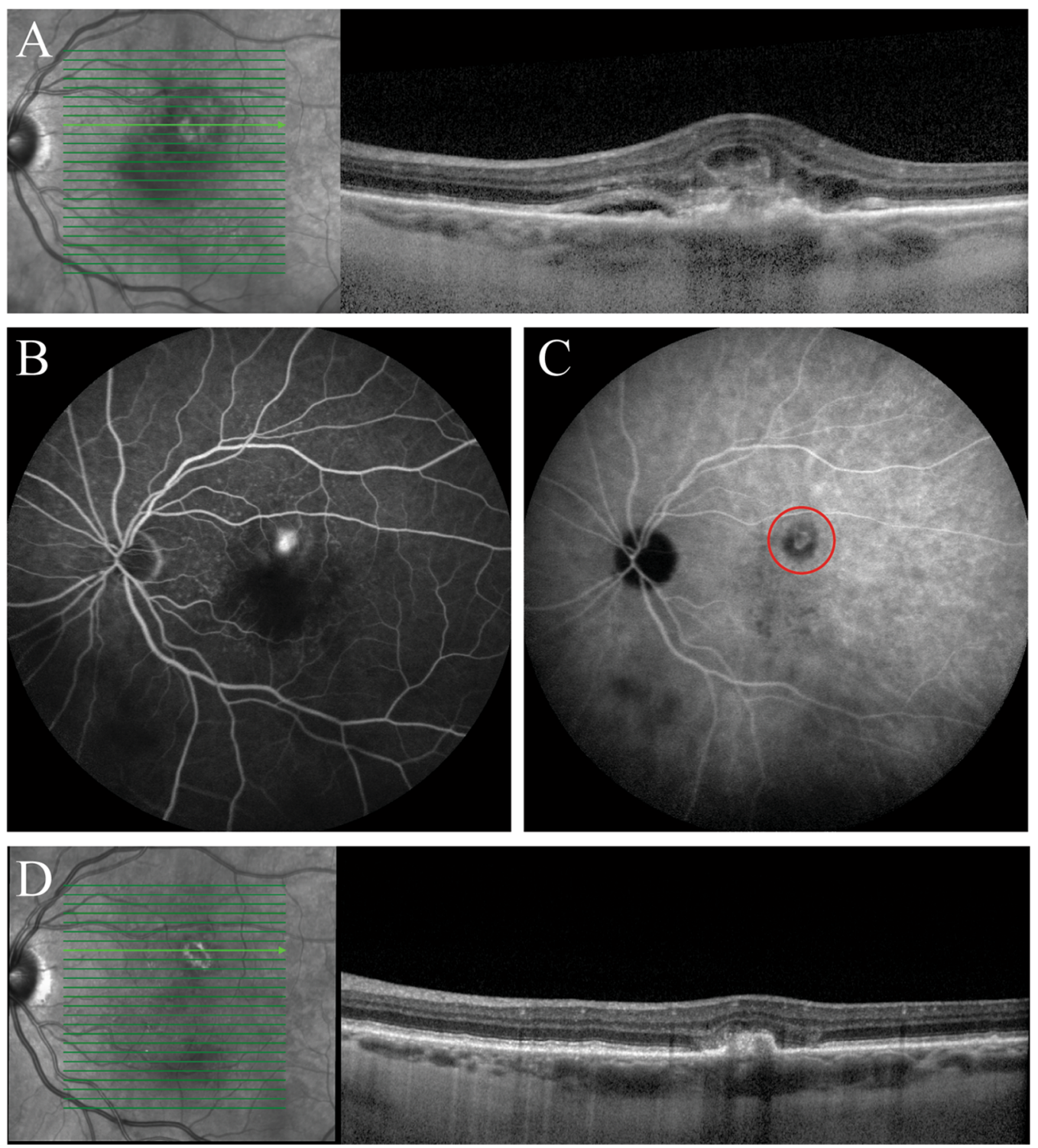

Fig. 2 Multimodal imaging of an eye of a 73-year-old man diagnosed with polypoidal choroidal vasculopathy who was treated with half-dose photodynamic therapy (PDT) and intravitreal anti-vascular endothelial growth factor (VEGF) injections. a, b Prior to initiation of half-dose PDT, a hyperreflective irregular retinal pigment epithelium layer with adjacent subretinal fluid highly suggestive of a polypoidal lesion was seen on optical coherence tomography (OCT) (a), and leakage of fluorescein was observed on fluorescein angiography (b). The patient had received 2

closure and fewest required injections [38, 39]. The EVEREST II study found that combination therapy of full-dose PDT and intravitreal intravitreal anti-VEGF injections before half-dose PDT. c The mild hyperfluorescent polypoidal lesion visible on indocyanine green angiography was targeted with half-dose PDT (within the red circle). $\mathbf{d}$ Compared to the imaging results before PDT, at the visit 6 months after half-dose PDT, the polypoidal lesion had regressed and the subretinal fluid had disappeared on OCT. This patient had received an additional 7 intravitreal anti-VEGF injections between half-dose PDT and the visit at 6 months after PDT

injections of ranibizumab was superior to monotherapy consisting of intravitreal injections of ranibizumab, in terms of BCVA and 
complete polyp regression [40]. However, recent studies suggest that anti-VEGF monotherapy with, for example, aflibercept has similar efficacy, at least in an Asian population [41, 42]. Since information reported in the literature is not clear with regard to the added efficacy of PDT in the treatment of PCV, we would consider the evidence of PDT in PCV to be of level 2 . To achieve polyp closure, the complete lesion, including both the polyps and the branching vascular networks visualized on ICGA, can be treated, but treatment can also be restricted to the polyps only (Fig. 2). In PCV patients, many clinicians perform PDT at the standard settings $[39,43]$, but half-dose PDT may also be considered [23]. No significant improvement in BCVA has been reported after PDT monotherapy [44]. To date, the literature suggests that the preferred treatment approach for PCV is either anti-VEGF monotherapy or anti-VEGF treatment combined with PDT $[45,46]$.

In our opinion, monotherapy consisting of intravitreal injections with anti-VEGF may be considered in PCV patients with relatively good BCVA as this treatment often leads to good visual and anatomical outcomes and has no risk of the occurrence of complications that can occur after PDT. When the clinician decides to perform PDT, full-settings PDT may be considered in PCV patients who had shown an insufficient response to reduced-settings PDT.

\section{Specific Considerations When Treating Choroidal Hemangioma}

PDT is considered to be the treatment of choice for choroidal hemangioma, as this treatment modality selectively targets abnormal choroidal vessels without damaging the overlying neurosensory retina. PDT is especially indicated in patients with either evidence of SRF or macular edema [47-51]. When the size of the tumor vessels exceeds the maximum diameter of the spot size that can be used during PDT, multiple treatment spots can be used in one session. For this purpose, a contact lens providing a wider field of view can be used, such as the Volk SuperQuad lens (magnification $\times 1.92$ ), or a
Volk TransEquator lens (magnification $\times 1.44$; both Volk Optical Inc.).

Three main PDT protocols have been described for the treatment of choroidal hemangioma, all of which use a standard (full) dose of $6 \mathrm{mg} / \mathrm{m}^{2}$, but vary in terms of additional settings:

- 'Standard' protocol: verteporfin is infused over a period of $10 \mathrm{~min}$; PDT treatment is $83 \mathrm{~s}$ (standard treatment time) at $600 \mathrm{~mW} /$ $\mathrm{cm}^{2}$ irradiance, starting exactly $15 \mathrm{~min}$ after the start of the verteporfin infusion, compatible with a standard fluence (radiant exposure) of $50 \mathrm{~J} / \mathrm{m}^{2}$ [48].

- 'Bolus' protocol: verteporfin is infused rapidly within $1 \mathrm{~min}$ (bolus); PDT treatment is $83 \mathrm{~s}$ (standard treatment time) at $600 \mathrm{~mW} /$ $\mathrm{cm}^{2}$ irradiance, starting exactly $6 \mathrm{~min}$ after the start of the verteporfin infusion, compatible with a standard fluence (radiant exposure) of $50 \mathrm{~J} / \mathrm{m}^{2}[49,50]$;

- 'High fluence' protocol: verteporfin is infused over a period of $10 \mathrm{~min}$; PDT treatment is $166 \mathrm{~s}$ (twice the treatment time of the aforementioned protocols) at $600 \mathrm{~mW} /$ $\mathrm{cm}^{2}$ irradiance, starting exactly $15 \mathrm{~min}$ after the start of the verteporfin infusion, compatible with an increased fluence (radiant exposure) of $100 \mathrm{~J} / \mathrm{m}^{2}$ [51].

Independent of tumor size, tumor controlwhich is usually defined as the elimination of exudation rather than complete tumor shrinkage-can be achieved in the vast majority of patients (Fig. 3) [52]. The latter two treatment protocols described in aforementioned text appear to have a higher single-treatment longterm success rate in terms of complete resolution of SRF [49-51]. The authors of one study reported that the visual outcome of patients with choroidal hemangioma increased from a mean visual acuity of 20/400 in the pre-PDT era to $20 / 63$ in the PDT era [53]. In addition, multiple case series in choroidal hemangioma patients found clinical improvements and minimal complications after PDT $[48,51,54,55]$. The level of evidence that is available for PDT in the treatment of choroidal hemangioma is considered to be level 2 due to the lack of large RCTs. Nevertheless, it is the 

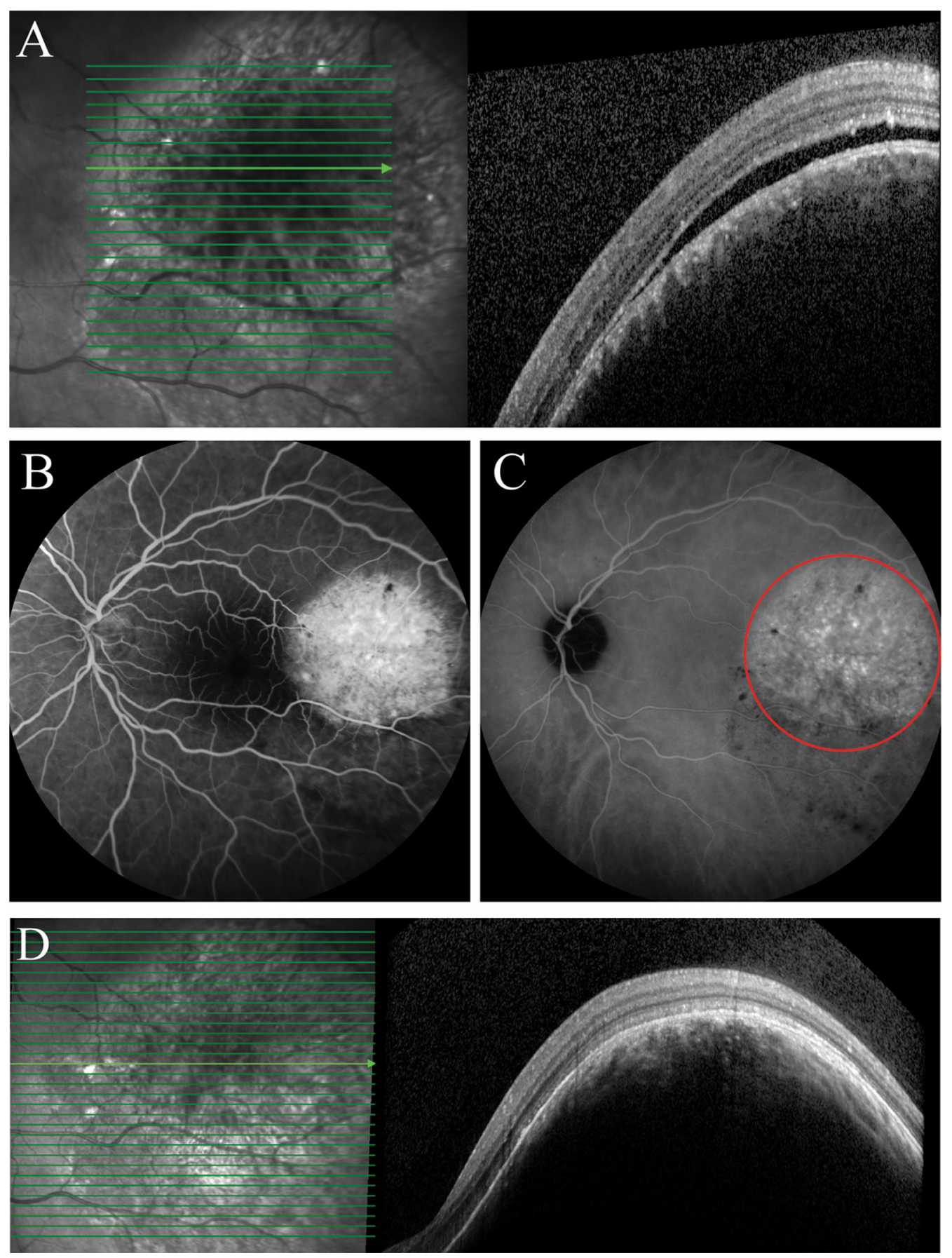

Fig. 3 Multimodal imaging of the an eye of a 51-year-old man diagnosed with choroidal hemangioma and treated with full-dose photodynamic therapy (PDT) according to the bolus protocol. a, b Prior to treatment, temporal to the macula an elevated lesion with subretinal fluid was visible on optical coherence tomography (OCT) (a), and diffuse leakage of fluorescein was observed on fluorescein angiography (b). c The hyperfluorescent area within the red circle on indocyanine green angiography was treated twice with full-dose PDT. d Compared to the imaging results before PDT, at the visit at 12 months after the last PDT, the choroidal hemangioma had regressed and subretinal fluid had disappeared on OCT 
preferred treatment for choroidal hemangioma since other interventions remain to be scrutinized.

\section{SAFETY}

\section{Contraindications}

The use of verteporfin is contraindicated when the patient shows hypersensitivity to either verteporfin or any component of the drug. In addition, patients with severe hepatic disease cannot receive PDT since severe hepatic impairment leads to a decrease in the metabolism of verteporfin into diacid. As the conversion of verteporfin is important to induce its effect, PDT is contraindicated in porphyria patients due to the absence of several enzymes in this patient population [56].

\section{Bilateral Treatment}

No studies have been performed on bilateral PDT on the same day. As transient visual complaints can occur after PDT, as well as irreversible vision loss due to chorioretinal atrophy in very rare cases, bilateral treatment should be performed with caution [57].

\section{Pregnancy and Postpartum Period}

Verteporfin has not been studied in pregnant women. In rats, the use of verteporfin has shown teratogenic effects. Verteporfin should only be used in pregnant women if the benefit justifies the potential risk to the fetus. Moreover, verteporfin and its metabolites have been detected in breast milk after full-dose PDT. As the effects of verteporfin on neonates are currently unknown, treatment should be postponed to the post-partum period, and breast feeding should be interrupted for at least $96 \mathrm{~h}$ after treatment $[56,58]$.

\section{Complications}

Only a few side effects have been reported during and after PDT. Severe complications, such as hypersensitivity reactions to the infusion, choroidal non-perfusion, and temporary retinal artery stenosis, have been described in a few cases and, consequently, patients have to be monitored during treatment $[18,56]$.

\section{Ocular Complications}

Transient visual complaints are the most frequently occurring ocular complication after PDT. These complaints, which has been described in up to $38 \%$ of patients, consist of various visual disturbances, such as abnormal vision and decreased vision, and generally occur within 1 week after PDT $[57,58]$. In one study, no significant differences on OCT and in retinal sensitivity on microperimetry were observed between patients who did and who did not experience these transient visual complaints, although transiently increased choroidal thickness and SRF accumulation were described [58]. Improvement or resolution of complaints was observed over time in the vast majority of these patients [59].

To minimize the risk of the occurrence of these severe ocular side effects, PDT at reduced settings (preferably using half of the standard verteporfin dose $\left(3 \mathrm{mg} / \mathrm{m}^{2}\right.$ instead of $\left.6 \mathrm{mg} / \mathrm{m}^{2}\right)$ is currently often used. In studies on reducedsettings PDT, no treatment-related ocular complications, such as a subretinal neovascularization or retinal pigment epithelium atrophy, have been observed [20,60,61].

\section{Systemic Complications}

Severe pain, inflammation, and blistering at the injection site can occur after extravasation of verteporfin. These side effects can be more pronounced when the affected area is exposed to light.

To prevent this complication, the largest possible arm vein has to be chosen for infusion of verteporfin, and the intravenous line has to be free-flowing before the verteporfin infusion is initiated [56]. Photosensitivity reaction is another important systemic complication, which usually occurs as a sunburn within $24 \mathrm{~h}$ after PDT in patients who do not take sufficient precautionary measures [56]. Lower back pain can also occur after PDT in up to $4 \%$ of patients. 
When these patients require a second treatment, this systemic adverse event can be effectively prevented by the intravenous administration of diclofenac shortly before treatment is initiated [62].

\section{IMPORTANT INFORMATION TO THE PATIENT}

Information to the patients should be provided well in advance because of two important aspects of PDT treatment. First, precautions need to be made to avoid sunlight after the treatment (sunglasses, scarf, hat, etc.). Second, following the treatment, use of motorized vehicles may be difficult due to transiently decreased vision, and is discouraged.

\section{Precautions to Avoid Sunlight}

Patients are photosensitive during the first $48 \mathrm{~h}$ after the administration of verteporfin and should therefore avoid exposing unprotected skin to sunlight. The duration of the period that exposure to sunlight should be avoided may be adjusted based on the verteporfin dose that was administered to the patient (e.g., 72 instead of $48 \mathrm{~h}$ when full-dose verteporfin was given). When treated patients have to go outside during the daytime within this 2-day period, it is recommended that they protect their skin and eyes by wearing protective skin-covering clothing and sunglasses. Patients may be encouraged to expose themselve to ambient indoor light in the early post-PDT period as this will help to eliminate the verteporfin more quickly by a process called photobleaching.

The verteporfin to be administered to a patient should not come into contact with light prior to its use in order to prevent a decrease in the treatment effect because light includes a wavelength that is similar to the $689-\mathrm{nm}$ wavelength of the laser that is used during treatment. This can be prevented by covering the verteporfin-filled syringe with, for example, aluminum foil. Moreover, direct contact of verteporfin with either eye or skin has to be avoided during treatment [56].

\section{Effects on Patient's Ability to Use Motorized Vehicles}

The necessary pharmacological pupil dilation that is achieved before PDT may impair vision for up to 1 day. In addition, patients may develop transient visual disturbances, such as decreased vision, in the post-PDT period. Therefore, the use of motorized vehicles is prohibited as long as these symptoms persist, which may be the case for up to several weeks [56].

\section{CONCLUSION}

In conclusion, in this article we describes practical approaches to PDT and highlight important practical aspects related to PDT. PDT plays an important role in the treatment of several chorioretinal diseases, such as CSC, PCV, and choroidal hemangioma. Level 1 evidence is available for half-dose PDT for the treatment of chronic CSC; consequently, half-dose (or halffluence) PDT is the treatment of choice for chronic CSC. The evidence for PCV and choroidal hemangioma is considered to be level 2 . For the treatment of PCV, anti-VEGF monotherapy or combination therapy of antiVEGF and PDT at standard or reduced settings can be considered. For the treatment of choroidal hemangioma, either standard treatment settings, a bolus protocol, or a high-fluence protocol can be used. Patients should be adequately informed of the need to avoid direct sunlight after treatment. Side effects during and after PDT are rare.

\section{ACKNOWLEDGEMENTS}

Funding. This study was supported by Stichting Macula Fonds; Retina Nederland Onderzoek Fonds; Stichting Blinden-Penning; Algemene Nederlandse Vereniging ter Voorkoming van Blindheid; Landelijke Stichting voor Blinden en Slechtzienden, which contributed through UitZicht (Delft, the Netherlands); Rotterdamse Stichting 
Blindenbelangen (Rotterdam, the Netherlands); Stichting Leids Oogheelkundig Ondersteuningsfonds (Leiden, the Netherlands); Haagse Stichting Blindenhulp (The Hague, the Netherlands); Stichting Ooglijders (Rotterdam, the Netherlands); the Gisela Thier Fellowship of Leiden University, Leiden, the Netherlands (CJFB); and the Netherlands Organisation for Scientific Research (VENI grant to CJFB). These funding organizations provided unrestricted grants and had no role in the design or conduct of this research. No funding or sponsorship was received for the publication of this article.

Authorship. All named authors meet the International Committee of Medical Journal Editors (ICMJE) criteria for authorship for this article, take responsibility for the integrity of the work as a whole, and have given their approval for this version to be published.

Disclosures. Elon H.C. van Dijk, Thomas J. van Rijssen, Yousif Subhi and Camiel J.F. Boon declare that no potential conflicts of interests exist in relation to this work.

Compliance with Ethical Guidelines. This article is based on previously conducted studies and does not contain any studies with human participants or animals performed by any of the authors.

Data Availability. Data sharing is not applicable to this article as no datasets were generated or analyzed during the current study.

Open Access. This article is licensed under a Creative Commons Attribution-NonCommercial 4.0 International License, which permits any non-commercial use, sharing, adaptation, distribution and reproduction in any medium or format, as long as you give appropriate credit to the original author(s) and the source, provide a link to the Creative Commons licence, and indicate if changes were made. The images or other third party material in this article are included in the article's Creative Commons licence, unless indicated otherwise in a credit line to the material. If material is not included in the article's Creative Commons licence and your intended use is not permitted by statutory regulation or exceeds the permitted use, you will need to obtain permission directly from the copyright holder. To view a copy of this licence, visit http://creativecommons.org/licenses/by$\mathrm{nc} / 4.0 /$.

\section{REFERENCES}

1. Epstein JH. Phototherapy and photochemotherapy. N Engl J Med. 1990;322(16):1149-51.

2. Daniell MD, Hill JS. A history of photodynamic therapy. Aust N Z J Surg. 1991;61(5):340-8.

3. Dolmans DE, Fukumura D, Jain RK. Photodynamic therapy for cancer. Nat Rev Cancer. 2003;3(5): $380-7$.

4. Schmidt-Erfurth U, Hasan T. Mechanisms of action of photodynamic therapy with verteporfin for the treatment of age-related macular degeneration. Surv Ophthalmol. 2000;45(3):195-214.

5. Dougherty TJ, Gomer CJ, Henderson BW, et al. Photodynamic therapy. J Natl Cancer Inst. 1998;90(12):889-905.

6. Bown SG. Science, medicine, and the future. New techniques in laser therapy, BMJ. 1998;316(7133): 754-7.

7. Newman DK. Photodynamic therapy: current role in the treatment of chorioretinal conditions. Eye (Lond). 2016;30(2):202-10.

8. Gomer CJ. Photodynamic therapy in the treatment of malignancies. Semin Hematol. 1989;26(1):27-34.

9. Henderson BW, Dougherty TJ. How does photodynamic therapy work? Photochem Photobiol. 1992;55(1):145-57.

10. Chan WM, Lam DS, Lai TY, Tam BS, Liu DT, Chan CK. Choroidal vascular remodelling in central serous chorioretinopathy after indocyanine green guided photodynamic therapy with verteporfin: a novel treatment at the primary disease level. $\mathrm{Br} \mathrm{J}$ Ophthalmol. 2003;87(12):1453-8.

11. Karim R, Sykakis E, Lightman S, Fraser-Bell S. Interventions for the treatment of uveitic macular edema: a systematic review and meta-analysis. Clin Ophthalmol. 2013;7:1109-44.

12. Shin JY, Woo SJ, Yu HG, Park KH. Comparison of efficacy and safety between half-fluence and full- 
fluence photodynamic therapy for chronic central serous chorioretinopathy. Retina. 2011;31(1): 119-26.

13. Alkin Z, Perente I, Ozkaya A, et al. Comparison of efficacy between low-fluence and half-dose verteporfin photodynamic therapy for chronic central serous chorioretinopathy. Clin Ophthalmol. 2014;8:685-90.

14. Nicolo M, Eandi CM, Alovisi C, et al. Half-fluence versus half-dose photodynamic therapy in chronic central serous chorioretinopathy. Am J Ophthalmol. 2014;157(5):1033-7.

15. Cheng CK, Chang CK, Peng CH. Comparison of photodynamic therapy using half-dose of verteporfin or half-fluence of laser light for the treatment of chronic central serous chorioretinopathy. Retina. 2017;37(2):325-33.

16. Zhao M, Zhang F, Chen Y, et al. A 50\% vs 30\% dose of verteporfin (photodynamic therapy) for acute central serous chorioretinopathy: one-year results of a randomized clinical trial. JAMA Ophthalmol. 2015;133(3):333-40.

17. Zhao MW, Zhou P, Xiao HX, et al. Photodynamic therapy for acute central serous chorioretinopathy: the safe effective lowest dose of verteporfin. Retina. 2009;29(8):1155-61.

18. van Rijssen TJ, van Dijk EHC, Yzer S, et al. Central serous chorioretinopathy: towards an evidencebased treatment guideline. Prog Retin Eye Res. 2019;73:100770.

19. Mohabati D, Hoyng CB, Yzer S, Boon CJF. Clinical characteristics and outcome of posterior cystoid macular degeneration in chronic central serous chorioretinopathy. Retina. 2019. doi: https://doi. org/10.1097/IAE.0000000000002683.

20. van Dijk EHC, Fauser S, Breukink MB, et al. Halfdose photodynamic therapy versus high-density subthreshold micropulse laser treatment in patients with chronic central serous chorioretinopathy: the place trial. Ophthalmology. 2018;125(10):1547-55.

21. Mohabati D, van Rijssen TJ, van Dijk EH, et al. Clinical characteristics and long-term visual outcome of severe phenotypes of chronic central serous chorioretinopathy. Clin Ophthalmol. 2018;12: 1061-70.

22. Breukink MB, Mohabati D, van Dijk EH, et al. Efficacy of photodynamic therapy in steroid-associated chronic central serous chorioretinopathy: a casecontrol study. Acta Ophthalmol. 2016;94(6): 565-72.
23. Wong IY, Shi X, Gangwani R, et al. One-year results of half- versus standard-dose photodynamic therapy combined with ranibizumab for polypoidal choroidal vasculopathy. Retina. 2018;38(4):725-30.

24. Yannuzzi LA, Slakter JS, Gross NE, et al. Indocyanine green angiography-guided photodynamic therapy for treatment of chronic central serous chorioretinopathy: a pilot study. Retina. 2003;23(3):288-98.

25. Cardillo Piccolino F, Eandi CM, Ventre L, Rigault de la Longrais RC, Grignolo FM. Photodynamic therapy for chronic central serous chorioretinopathy. Retina. 2003;23(6):752-63.

26. Lai TY, Chan WM, Li H, Lai RY, Liu DT, Lam DS. Safety enhanced photodynamic therapy with half dose verteporfin for chronic central serous chorioretinopathy: a short term pilot study. Br J Ophthalmol. 2006;90(7):869-74.

27. Taban M, Boyer DS, Thomas EL, Taban M. Chronic central serous chorioretinopathy: photodynamic therapy. Am J Ophthalmol. 2004;137(6):1073-80.

28. Ruiz-Moreno JM, Lugo FL, Armada F, et al. Photodynamic therapy for chronic central serous chorioretinopathy. Acta Ophthalmol. 2010;88(3):371-6.

29. van Rijssen TJ, van Dijk EHC, Dijkman G, Boon CJF. Clinical characteristics of chronic central serous chorioretinopathy patients with insufficient response to reduced-settings photodynamic therapy. Graefes Arch Clin Exp Ophthalmol. 2018;256(8):1395-402.

30. Haga F, Maruko R, Sato C, Kataoka K, Ito Y, Terasaki H. Long-term prognostic factors of chronic central serous chorioretinopathy after half-dose photodynamic therapy: a 3-year follow-up study. PLoS ONE. 2017;12(7):e0181479.

31. Lotery A, Sivaprasad S, O'Connell A, et al. Eplerenone for chronic central serous chorioretinopathy in patients with active, previously untreated disease for more than 4 months (VICI): a randomised, double-blind, placebo-controlled trial. Lancet. 2020;395(10220):294-303.

32. Wang MS, Sander B, Larsen M. Retinal atrophy in idiopathic central serous chorioretinopathy. Am J Ophthalmol. 2002;133(6):787-93.

33. Lai FH, Ng DS, Bakthavatsalam M, et al. A multicenter study on the long-term outcomes of halfdose photodynamic therapy in chronic central serous chorioretinopathy. Am J Ophthalmol. 2016;170:91-9.

34. Nicolo M, Zoli D, Musolino M, Traverso CE. Association between the efficacy of half-dose 
photodynamic therapy with indocyanine green angiography and optical coherence tomography findings in the treatment of central serous chorioretinopathy. Am J Ophthalmol. 2012;153(3): 474-80.e1.

35. Piccolino FC, De La Longrais RR, Manea M, Cicinelli S. Posterior cystoid retinal degeneration in central serous chorioretinopathy. Retina. 2008;28(7): 1008-122.

36. Chung CY, Chan YY, Li KKW. Angiographic and tomographic prognostic factors of chronic central serous chorioretinopathy treated with half-dose photodynamic therapy. Ophthalmologica. 2018;240(1):37-44.

37. van Rijssen TJ, van Dijk EHC, Dijkman G, Boon CJF. Clinical characteristics of chronic central serous chorioretinopathy patients with insufficient response to reduced-settings photodynamic therapy. Graefes Arch Clin Exp Ophthalmol. 2018;256(8):1395-1402.

38. Koh AH, Chen LJ, Chen SJ, et al. Polypoidal choroidal vasculopathy: evidence-based guidelines for clinical diagnosis and treatment. Retina. 2013;33(4):686-716.

39. Wong RL, Lai TY. Polypoidal choroidal vasculopathy: an update on therapeutic approaches. J Ophthalmic Vis Res. 2013;8(4):359-71.

40. Koh A, Lai TYY, Takahashi K, et al. Efficacy and safety of ranibizumab with or without verteporfin photodynamic therapy for polypoidal choroidal vasculopathy: a randomized clinical trial. JAMA Ophthalmol. 2017;135(11):1206-13.

41. Lee WK, Iida T, Ogura Y, et al. Efficacy and safety of intravitreal aflibercept for polypoidal choroidal vasculopathy in the planet study: a randomized clinical trial. JAMA Ophthalmol. 2018;136(7): 786-93.

42. Farooq A, Frazier H, Marcus WB, Fechter C, Singh $\mathrm{H}$, Marcus DM. Intravitreal aflibercept for neovascular polypoidal choroidal vasculopathy in a predominantly non-Asian population: rival results. Ophthalmic Surg Lasers Imaging Retina. 2017;48(1):34-52.

43. Lai TY, Chan WM. An update in laser and pharmaceutical treatment for polypoidal choroidal vasculopathy. Asia Pac J Ophthalmol (Phila). 2012;1(2):97-104.

44. Oishi A, Kojima H, Mandai M, et al. Comparison of the effect of ranibizumab and verteporfin for polypoidal choroidal vasculopathy: 12-month laptop study results. Am J Ophthalmol. 2013;156(4): 644-51.
45. Palkar AH, Khetan V. Polypoidal choroidal vasculopathy: an update on current management and review of literature. Taiwan $\mathrm{J}$ Ophthalmol. 2019;9(2):72-92.

46. Doble B, Finkelstein EA, Tian Y, et al. Cost-effectiveness of intravitreal ranibizumab with verteporfin photodynamic therapy compared with ranibizumab monotherapy for patients with polypoidal choroidal vasculopathy. JAMA Ophthalmol. 2020. https://doi.org/10.1001/jamaophthalmol. 2019.5628 .

47. Singh AD, Kaiser PK, Sears JE, Gupta M, Rundle PA, Rennie IG. Photodynamic therapy of circumscribed choroidal haemangioma. $\mathrm{Br} \mathrm{J}$ Ophthalmol. 2004;88(11):1414-8.

48. Boixadera A, Garcia-Arumi J, Martinez-Castillo V, et al. Prospective clinical trial evaluating the efficacy of photodynamic therapy for symptomatic circumscribed choroidal hemangioma. Ophthalmology. 2009;116(1):100-5.e1.

49. Verbraak FD, Schlingemann RO, de Smet MD, Keunen JE. Single spot PDT in patients with circumscribed choroidal haemangioma and near normal visual acuity. Graefes Arch Clin Exp Ophthalmol. 2006;244(9):1178-82.

50. Verbraak FD, Schlingemann RO, Keunen JE, de Smet MD. Longstanding symptomatic choroidal hemangioma managed with limited PDT as initial or salvage therapy. Graefes Arch Clin Exp Ophthalmol. 2003;241(11):891-8.

51. Blasi MA, Tiberti AC, Scupola A, et al. Photodynamic therapy with verteporfin for symptomatic circumscribed choroidal hemangioma: five-year outcomes. Ophthalmology. 2010;117(8):1630-7.

52. Schmidt-Erfurth UM, Michels S, Kusserow C, Jurklies B, Augustin AJ. Photodynamic therapy for symptomatic choroidal hemangioma: visual and anatomic results. Ophthalmology. 2002;109(12): 2284-94.

53. Shields CL, Dalvin LA, Lim LS, et al. Circumscribed choroidal hemangioma: visual outcome in the prephotodynamic therapy era versus photodynamic therapy era in 458 cases. Ophthalmol Retina. 2020;4(1):100-10.

54. Jurklies B, Anastassiou G, Ortmans S, et al. Photodynamic therapy using verteporfin in circumscribed choroidal haemangioma. Br J Ophthalmol. 2003;87(1):84-9.

55. Porrini G, Giovannini A, Amato G, Ioni A, Pantanetti M. Photodynamic therapy of circumscribed choroidal hemangioma. Ophthalmology. 2003;110(4):674-80. 
56. Investigator's Brochure: Verteporfin for injection (Visudyne ${ }^{\circledR}$ ) for use in photodynamic therapy (PDT) for ocular studies. 2008.

57. Azab M, Benchaboune M, Blinder KJ, et al. Verteporfin therapy of subfoveal choroidal neovascularization in age-related macular degeneration: metaanalysis of 2-year safety results in three randomized clinical trials: treatment of age-related macular degeneration with photodynamic therapy and verteporfin in photodynamic therapy study report no. 4. Retina. 2004;24(1):1-12.

58. van Dijk EHC, Dijkman G, Theelen T, Hoyng CB, Boon CJF. Short-term findings on optical coherence tomography and microperimetry in chronic central serous chorioretinopathy patients treated with halfdose photodynamic therapy. Retin Cases Brief Rep. 2018;12(4):266-71.
59. Arnold JJ, Blinder KJ, Bressler NM, et al. Acute severe visual acuity decrease after photodynamic therapy with verteporfin: case reports from randomized clinical trials-TAP and VIP report no. 3 . Am J Ophthalmol. 2004;137(4):683-96.

60. Liu CF, Chen LJ, Tsai SH, et al. Half-dose verteporfin combined with half-fluence photodynamic therapy for chronic central serous chorioretinopathy. J Ocul Pharmacol Ther. 2014;30(5):400-5.

61. Tseng CC, Chen SN. Long-term efficacy of half-dose photodynamic therapy on chronic central serous chorioretinopathy. Br J Ophthalmol. 2015;99(8): 1070-7.

62. Theelen T, Hoyng CB. Intravenous diclofenac as prophylactic treatment for verteporfin-associated low back pain. Eur J Ophthalmol. 2008;18(5):805-8. 